\title{
Research on an Improved RFID Anti-collision Algorithm in the Internet of Things
}

\author{
Jiangang Jin \\ (Software Technology Vocational College, North China University of Water \\ Resources and Electric Power, Zhengzhou 450045, China) \\ henanjjg@sina.com
}

\begin{abstract}
In the RFID system communication of Internet of things, the tag and the reader exchange data easy to collide, which makes the tag can be identified. Based on the study on the basis of binary arithmetic, by the collision process of the instruction set different status to lock collision bits, and these sample records locked position, then the paging process for sample collection the remaining collision bit in descending order of selection process, and achieved good results. Through the algorithm search times, communication capacity and throughput of index analysis, compared with the traditional binary collision algorithm has been significantly improved, to improve the efficiency of the system.
\end{abstract}

Keywords: RFID, Anti-collision, Lock-bit, Sample

\section{Introduction}

RFID (Fadio Frequeency Identification) is a kind of technology that can get data from the contact without contact. The basic principle of it is to rely on electromagnetic waves as the transmission medium to carry out two-way communication, achieve the purpose of automatic recognition [1]. This technique has strong automatic identification, quick response, hardware cost low and long life and other advantages and is widely used in medical, smart card, attendance system, and project tracking in.

RFID is mainly composed of the reader, tag and processing system. Its working principle has unique identifier ID to enter in the field range of the reader, through the wireless network with the label of two-way communication, so as to realize the label of data acquisition. To achieve data processing and resource sharing. But the acquisition has an obvious disadvantage is the presence of range and multiple tags into the reader, it will produce the collision with the communication between readers, leading to the label cannot be identified. At present, common tag anti-collision algorithm is based on the time division multiplex of non-determination algorithm and deterministic algorithm, non-deterministic algorithm is on behalf of the Aloha algorithm [2]. The algorithm is simple and easy to implement, can meet the basic requirements of the occasion and read only labels [3]; Deterministic algorithm is mainly represented by binary search algorithm [4]. The algorithm needs to search many times, the amount of data is relatively large, and the throughput is low. Domestic and foreign scholars have made a research on this, and have put forward different solutions. Document 5 proposed an improved RFID tag anti-collision algorithm, RFID algorithm is improved, to establish a new RFID module, improve the efficiency of RFID system identification. Document 6 made an optimization of anti-collision algorithm. The algorithm constructs new request establishment, by the recognition by double-digit collision arbitration, greatly reducing the collision detection when corresponding tag number, so as to reduce the collision 
probability. Document 7 proposed using with the characteristics of the label sequence number of the uniqueness and Manchester code identification tag collision position and the binary optimization code, to shorten the length of the transmitted symbols.

Based on the study on the basis of binary arithmetic, by the collision process of the instruction set different status to lock collision bits, and these sample records locked position, then the paging process for sample collection the remaining collision bit in descending order of selection process, and achieved good results. Algorithm experimental search times, communication capacity and throughput of index analysis, compared with the traditional binary collision algorithm has been significantly improved, to improve the efficiency of the system.

\section{Basic Binary Anti-collision Search Algorithm}

\subsection{Algorithm Process}

Binary search anti-collision algorithm mainly uses bit collision detection protocol as an approach to prevent collisions, this scheme requires the reader can accurately obtain the bit positions of the collision. It is generally the Manchester coding is used in multiple tags at the same time, the response time to identify a collision position according to the position of the collision according to certain rules to search out the tag. Assuming that the length of the tag ID is $N$, the bit is represented as $[N, N-1, N-2, \cdots 1]$, divided into the following three steps:

Step1: Initialization. Reader first send the instruction to the serial number is 1 command, then all tags will put their ID to send to the reader. The reader ID number were detected collision position hypothesis (assuming the position in $X$ ), according to the position, readers get next request command sequence number parameter list. The sequence number of the former $(N-X)$ position and the corresponding ID number of the former $(N-X)$ the same, the first $X$ position is 0 , the rest of the $(X-1)$ is 1 .

Step2: Each tag ID number according to the reader in step 1 of the new sequence number are compared, if less than or equal to the sequence number of the tag will be sent back to the reader. Otherwise, it is not to do any treatment.

Step3: Repeat step 2, until only a tag response process ended, recognize the label after the go to choice, and then executing step 1, until all the tags are identified.

\subsection{Algorithm Efficiency Analysis}

Reader sends the command request length is mainly its code length, length of related parameters and tag response command data length and hence binary algorithm to accept feedback command number are as follows, binary search algorithm to search element to the first label issued order number:

$L(Q)=l b(Q)+1$

And then after the first label to go after the selection, again for the first search. At this time waiting for the identification tag is $Q-i-1$, followed by analogy to wait for the identification of the tag is $Q-i-1$. Therefore, the number of commands in the $i$ tag is:

$L(Q-i-1)=l b(Q-i-1)+1$

(2) 
The number of times required to complete all the tags is:

$$
T=\sum_{i=1}^{Q}(l b(i)+1)
$$

(3)

So it can be found, efficiency of the binary collision algorithm is mainly electronic tag response times and response times are longer, the number of complete all label more. This will reduce the efficiency of the algorithm, to occupy more communication time.

\section{Improved Binary RFID Algorithm}

\subsection{Raise Question}

Assuming that all the electronic tag reader within the scope of the work area are in a state response to reply, if there is a position of the electronic tag $k$ conflict, then the binary bits for this $k$ cannot be identified, the reader and electronic tags the process of dialogue between the content of the dialogue is to follow the entire sequence, so that the presence of some of the information will be a lot of extra presence. Therefore, for the improvement of the binary algorithm is mainly from the redundant information in the sequence of the reduction and in other locations for the treatment of anti - collision, so as to improve the efficiency of the algorithm.

\subsection{Improved Binary Algorithm Related Instructions}

The specific meaning of each instruction is as follows:

REQUEST(ID,1) Lock page: ID said reader to send the first instruction sequence obtained after the sequence number, the reader was used to determine the data collision accurate bit position after, one-time extraction of multiple, conflicting location information and all set to 1 , other non-collision position is set to 0 , using the standard a new sequence. In response, electronic tags will be their ID data bits and the reader in a serial number, will set the position of the bit 1 corresponding to the label lock returned to the reader.

SELECT(ID): The reader uses the serial number as a parameter to send it to an electronic tag. An electronic tag with the same serial number to execute the command. Other serial number of electronic tags only to the REQUEST command response.

READ: The electronic tag is selected to send the data to the reader.

UNSELECT: Set the electronic tag to enter the silent state, to receive the REQUEST command does not respond.

\subsection{Improved Binary Algorithm Steps}

Step1: Initialization of the reader commands and related parameters settings

Step2: Reader transmits a REQUEST ( $X$ ) command, the label will of its own ID code value and the reader sends out the results are compared, both results if less than or equal to, labels will send out his ID, otherwise not.

Step3: Reader will continue to search around the tags send signal, if you don't get a response from electronic label, so turn step 2, reader will continue to send commands; otherwise received signals go to step 4 . 
Step4: Reader to reply to the electronic tag to make response signal decoding, by comparing results of both the decoding to judge whether a collision has occurred. If no collision is sent select and read command of read and write operations, and then issued a command UNSELECT, make the label into safety state.

Step5: After the collision of step 4. How soon found the specific location of the collision is key to the improved algorithm, set the responding tags for three states: wait state, a dormant state, and exposed, and set a state counter. When the counter state values for 0 that the label in the wait command status, greater than 0 is illustrated in a dormant state, exposed that reader has been identified by the label, no longer responds to signals emitted by the reader. Reader in collision detection when first collision detection position, assumed to be in the $X$ bit, then a send command sequence for $(N-X)$, reader REQUEST $(I D, X)$ to send commands, label after receiving this command tag comparison ID will occur collision $X$ bit set into the locked state, check the lock for locating the highest for 1 label for this command to make the response and the label in waiting for the order to the state, otherwise enter a state of dormancy. Reader sends a select and a read instruction. On the label of read and write operations, the reader to send command UNSELECT, issue the command, the label set is exposed. Setting this happens Sample sample collection, record the results on $X$ bit of $\left(S_{x}, i\right)$, where $i$ is the recording position in the sequence, followed by recording the collision position and the formation of a collection of $\left\{\left(S_{x}, i\right)\left(S_{y}, j\right) \ldots . .\left(S_{n}, m\right)\right\}$, and then follow the sample set corresponding to the position of the collision position in the sequence the strategic choice in descending identification, constantly repeat the operation until all the tag identification lock collision bits 0 and 1 in the sequence is completed, go to step 6 .

Step6: All the electronic tag locations identified, the end of the process.

\section{Algorithm Analysis}

To measure the RFID anti-collision algorithm performance mainly from the number of search, communication capacity and throughput aspects, through carries on the comparative analysis with binary collision algorithm representative algorithm in BS algorithm and DBS algorithm.

\subsection{Search Times}

Reader role within the set range, with an electronic tag as $N, X$ is the number of bits for the collision, $S$ is the number of search elements Reader occurred. BS algorithm search for the number of formula (4), the DBS algorithm searches for formula (5), the algorithm searches for formula (6). The comparison results are shown in Figure 3.

$S 1=\log _{2} N+1$

$S 2=N+1$

$$
S 3=2^{N}+1
$$




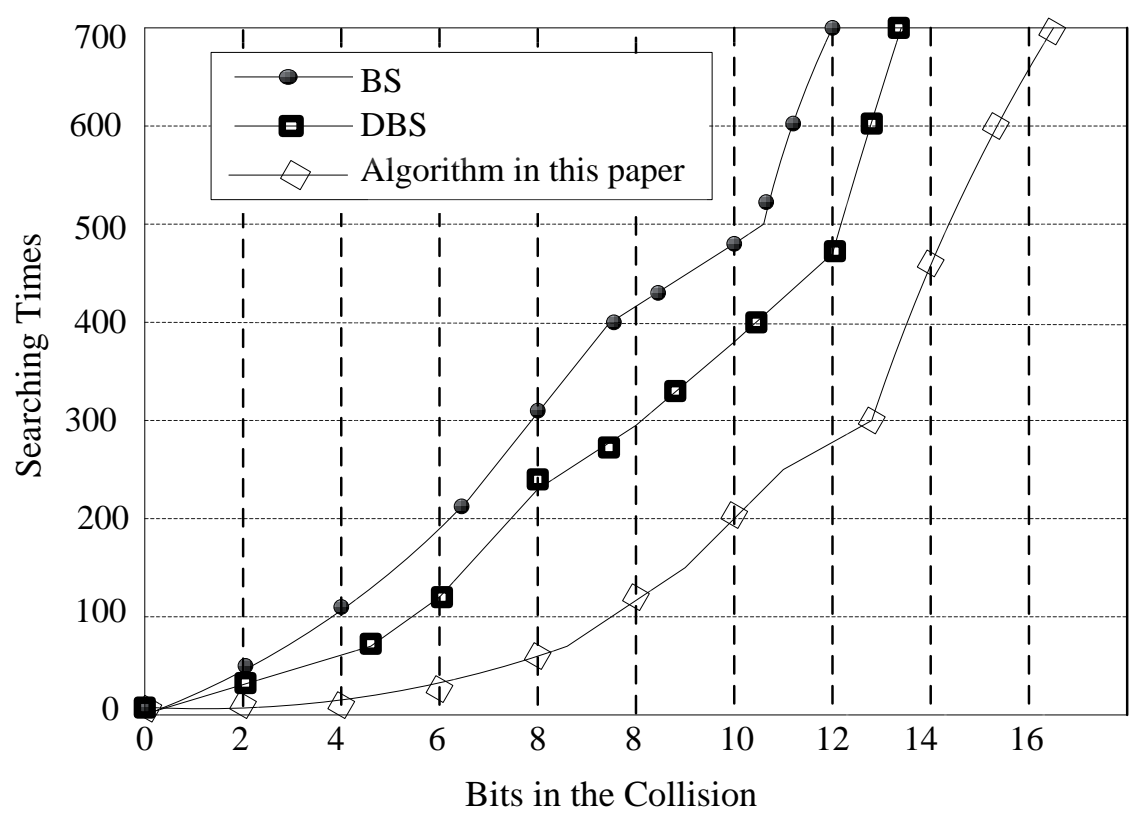

Figure 1. Relationship between Searching Times and Collision

From Figure 3 can be found, the algorithm in the number of search compared with BS, DBS algorithm has greatly reduced, when the collision position more, the more obvious the effect, when a collision is a 14 bit, the effect is obvious.

\subsection{Bit Rate of Transmission Data Communication}

In order to further illustrate the superiority of the algorithm proposed in this paper. This paper only studies the reader communication data bits, mainly because the reader sends traffic bit number and label to return the data communication of equal length. The serial number label length set to $M$, the BS algorithm complete search $N$ labels on the transmission of data communication for data length formula (6), the length of data communication in DBS algorithm need for formula (7), the search algorithm is divided into two parts, a lock is "0" and "1" of the binary code, the second is for collision bits to encode the binary code, so the length of the data communication for equation (8). Set the number of tags for the range of $[2,16]$, compare the results shown in Figure 4 and Table 1.

$L 1=M \times\left(\log _{2} N+1\right)$

$L 2=M \times(N+1)$

(8)

$L 3=(M 1+M 2) \times\left(2^{N}+1\right)$

(9) 


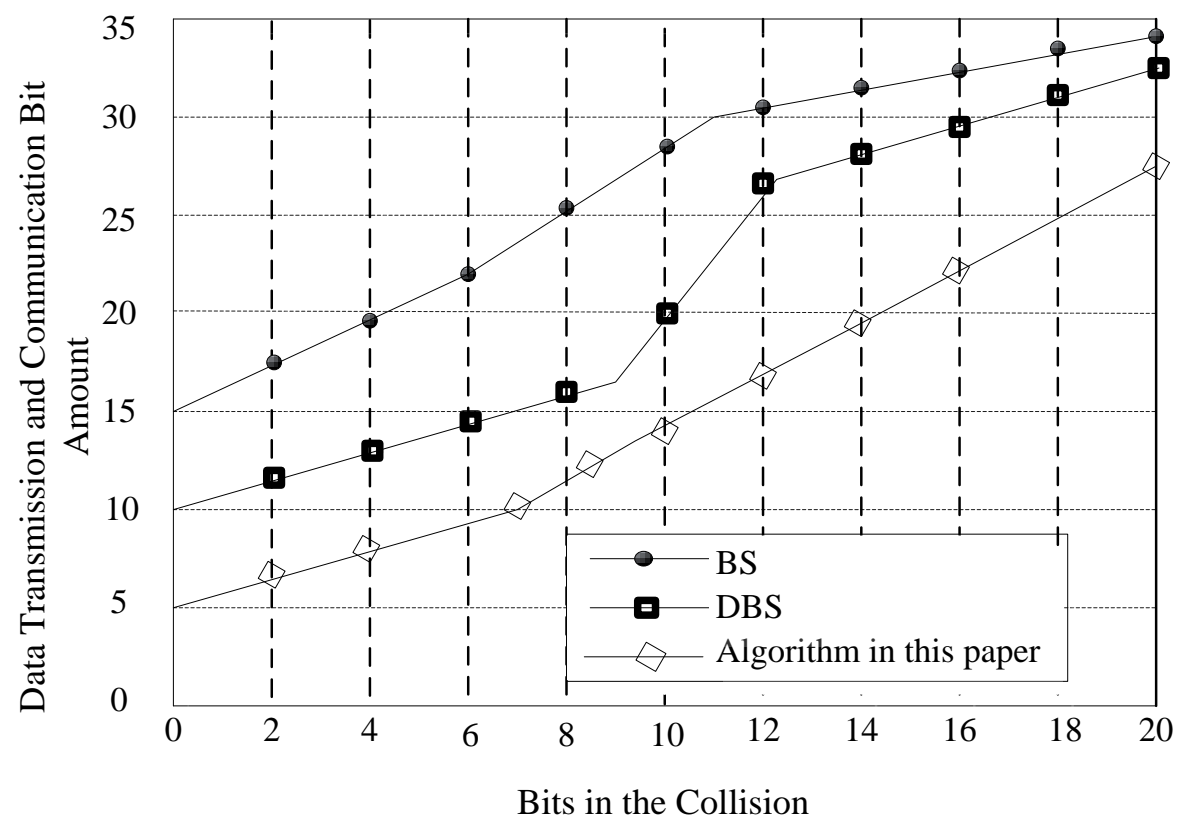

Figure 2. Relationship between Data Transmission and Communication Bit Amount and Collision Times

Table 1. Communication Bit Amount of Three Algorithm Readers

\begin{tabular}{c|c|c|c}
\hline Tag Number & BS Algorithm & DBS Algorithm & $\begin{array}{c}\text { Algorithm in this } \\
\text { paper }\end{array}$ \\
\hline 2 & 4278 & 3187 & 2105 \\
4 & 5000 & 3500 & 3000 \\
8 & 6000 & 4125 & 3381 \\
16 & 7500 & 7000 & 5245 \\
\hline
\end{tabular}

From Figure 4 and Table 1 can be found, BS and DBS algorithm consume amount of data in the communication with more labels data consumption is large, but the algorithm in this paper consumption is relatively small and the expenditure of stable, indicating that the algorithm is stable.

\subsection{Throughput}

Throughput relates to data in the process of identification data transmission efficiency, set $N$ search tags, the BS algorithm complete search system throughput formula (10), DBS algorithm search throughput formula (11), the algorithm to complete throughput formula (12), comparing the results as shown in Figure 5.

$$
\lambda 1=\frac{N}{\log _{2} N+1}
$$

$\lambda 2=\frac{N}{N+1}$ 
(11)

$\lambda 3=\frac{N}{2^{N}+1}$

(12)

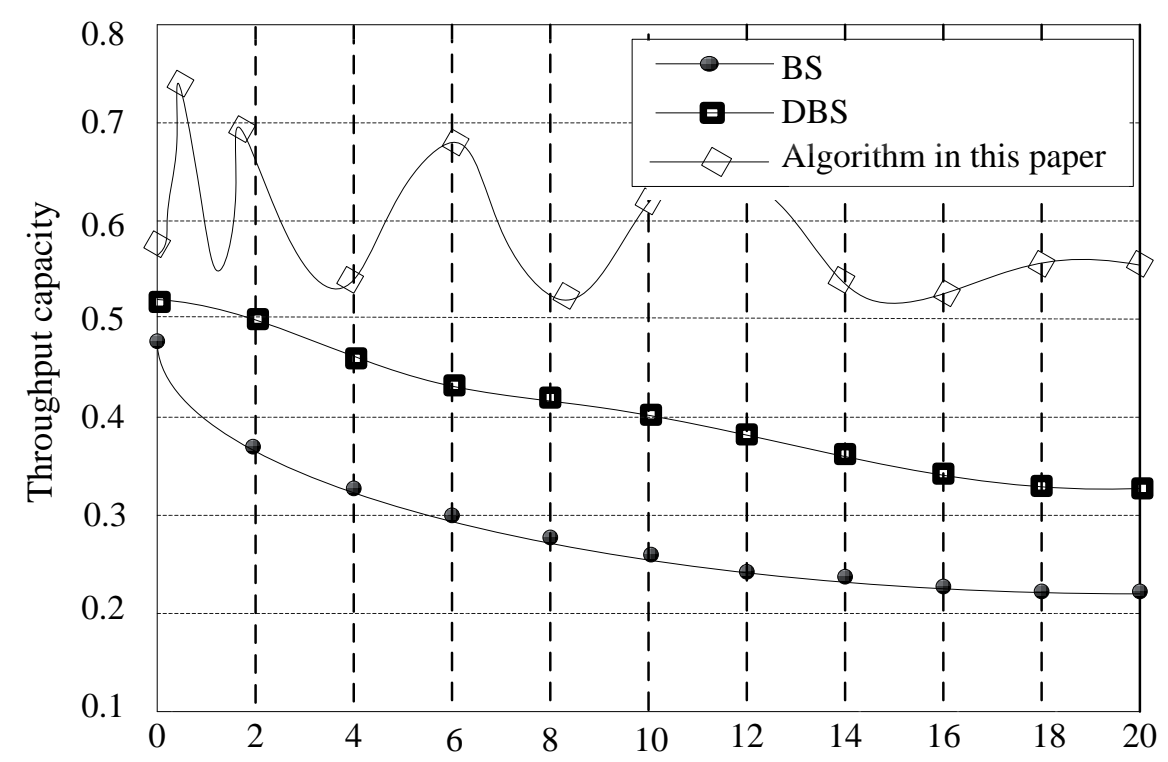

Bits in the Collision

\section{Figure 3. Comparison of Relationship between Throughput and Collision Times for Different Algorithms}

It can be found in Figure 5, the algorithm throughput presented wavy change and most always outperforms the BS algorithm and DBS algorithm, especially when the number of tags is becoming larger and more obviously see the superiority of the algorithm.

\section{Conclusion}

Based on the study on the basis of binary arithmetic, by the collision process of the instruction set different status to lock collision bits, and these sample records locked position, then the paging process for sample collection the remaining collision bit in descending order of selection process, and achieved good results. Algorithm experimental search times, communication capacity and throughput of index analysis, compared with the traditional binary collision algorithm has been significantly improved, to improve the efficiency of the system.

\section{References}

[1] Finkenzeller K., "RFID-Handbook,Fundamentals and Applications in Contactless Smart Cards and Identificaion[M]", New Jersey, John Wiley\&Sons Ltd, (2003).

[2] Peng Q. S., Zhang M. and Wu W. M., "Variant enhanced dynamic framed slotted ALOHA algorithm for fast object identificaion in RFID system[C]//Anti-Counterfeitingm Security, Identificaion, 2007 IEEE International Workshop, (2007), pp. 88-91.

[3] Ryu J., Lee H. and Seok Y., “Ahybrid query tree protocol for tag collision arbitration in RFID 
systems[C]",// Proceedings of IEEE International Conference on Communicaions Glasgow, Scotland: IEEE, (2007), pp. 5981-5986.

[4] Kim Y. H. and Kim S. S., "Improved 4-ary query tree algorithm for anticollision in RFID system[C]",// International Conference on Advanced Information Networking and Applicaions, Bradford, United Kingdom, (2008), pp. 699-704.

[5] Shi W.-D., “An Improved RFID Label Anti Collision Algorithm[J]”, Bulletin of Science and Technology, (2015), vol. 31, no. 4, pp. 121-123.

[6] Shi J.-Y., "An Optimized Search Algorithm Based on the Binary for RFID Tags Collision[J]”, Journal of Inner Mongolia Normal University (Natural Science Edition), vol. 44, no. 2, (2015), pp. 249-253.

[7] Cao J., "Research on a binary search rfid anti-collision algorithm with optimised coding[J]", Computer Applications and Software, vol. 32, no. 6, (2015), pp. 283-286.

[8] Wang X., "Research on RFID anti-collision algorithms based on binary tree[J]", Journal on communications, vol. 31, no. 6, (2010), pp. 49-5.

\section{Authors}

Jiangang Jin (1972.11-) Lecturer, Master, Research Orientation: Computing Network; 